\title{
Effect of nitrogen impurities on ZnS polymorphism
}

\author{
I. S. Popov, A. S. Vorokh, A. N. Enyashin \\ Institute of Solid State Chemistry UB RAS, Ekaterinburg, Russia \\ popov@ihim.uran.ru
}

PACS 61.72.Vv, 61.72.Bb, 31.15.E-

DOI 10.17586/2220-8054-2019-10-1-86-91

\begin{abstract}
The ZnS polymorphs - sphalerite and wurtzite - have the very close formation energies, setting their coexistence in nature. Moreover, numerous cases of a disordered phase formation based on these polymorphs have been registered. However, sphalerite is a common mineral, while wurtzite is rare. Perhaps the wider distribution of sphalerite can be explained by means of stabilizing effect from impurities. In this paper, the most stable form and the localization of nitrogen impurities in both $\mathrm{ZnS}$ polymorphs is screened using the methods of quantum chemistry. The influence of impurity on polymorphic wurtzite-sphalerite equilibrium is disclosed. According to the obtained results, the introduction of nitrogen impurities facilitates the domination of sphalerite over wurtzite.
\end{abstract}

Keywords: nitrogen impurity, $\mathrm{ZnS}$ polymorphism, polymorphic equilibria, $\mathrm{ZnS}$ nitridation, MD simulations.

Received: 27 December 2018

Revised: 19 January 2019

\section{Introduction}

In natural deposits, zinc sulfide occurs in the form of two polymorphs: sphalerite and wurtzite. Sphalerite is a more common mineral, while wurtzite is less frequent. Sphalerite is also predominant in $\mathrm{ZnS}$ of biogenic origin $[1,2]$. Wurtzite is a high-temperature phase formed from sphalerite when heated to $1020{ }^{\circ} \mathrm{C}$ [3]. The phase transition temperature can be significantly reduced by reducing the size of $\mathrm{ZnS}$ samples to nanoscale [4] or bringing sphalerite nanoparticles into contact with wurtzite-like $\mathrm{ZnO}$ nanoparticles [5]. According to theoretical estimations, the difference in the formation energies between wurtzite and sphalerite $\mathrm{ZnS}$ is negligibly small ( $3.25 \mathrm{meV} /$ atom) [6]. Both phases can be obtained with different degree of phase purity in the lab, depending on the conditions of synthesis [7-9]. In addition, there are examples of the formation of numerous intermediate polytypes and disordered structures, which can be derived from the wurtzite and sphalerite polymorphs [10]. The structures of disordered phases can be described as violations of the $\mathrm{ZnS}$ layers' alternation along the $c$ direction of the hexagonal wurtzite lattice. The phase of wurtzite corresponds to the layers' alternation $\mathrm{A}-\mathrm{B}-\mathrm{A}-\mathrm{B}-\mathrm{A}-\mathrm{B}$ along vector $c$, while sphalerite is formed by alternating $\mathrm{A}-\mathrm{B}-\mathrm{C}-\mathrm{A}-\mathrm{B}-\mathrm{C}$.

All factors affecting the formation of a particular $\mathrm{ZnS}$ phase in a solvent medium have not been fully identified, yet. The addition of the tetrabutylammonium hydroxide modifier to the alcohol solution leads to the formation of wurtzite, whereas sphalerite was mainly formed without the modifier [9]. This result was attributed by the authors [9] to the higher modifier adsorption energy on the crystal seed of the wurtzite phase. A mechanism for the $\mathrm{ZnS}$ crystal seed formation in water was proposed, relaying on the molecular mechanics calculations and suggesting a higher stability of sphalerite seeds [11]. Other molecular dynamics studies show that wurtzite $\mathrm{ZnS}$ in vacuum is more stable in a nanoscale form [12], while wetting the surface with water leads to stabilization of sphalerite. A previous study [2] assumes that the interaction between the surface of biogenic ZnS nanoparticles with some peptides may contribute to the formation of a crystal with fewer defects due to a decrease in the polarity of the ZnS crystal surface. All these facts indicate a significant role of surface interactions in the formation of a $\mathrm{ZnS}$ crystalline structure of one or another type.

Impurities within the bulk are another factor that may have a significant impact on the stacking order of the $\mathrm{ZnS}$ layers. It was assumed in the past that under normal conditions another $\mathrm{ZnS}$ polymorph (matraite) could form in addition to sphalerite and wurtzite [13]. Currently, the matraite is identified as (111)-twined sphalerite [14], which twinning is likely forced by the admixtures of $\mathrm{Fe}, \mathrm{Mn}, \mathrm{Cu}$, and $\mathrm{In}$ - the elements proned to accumulation in (111) plane of growing sphalerite. The role of $\mathrm{Cu}$ and In admixtures in $\mathrm{ZnS}$ was investigated [15]. It was shown that the concentration of these elements is higher in sphalerite than in wurtzite. Presumably, a higher concentration of $\mathrm{Cu}$ and $\mathrm{In}$ in the cubic lattice of sphalerite is associated with a greater symmetry similarity between the sphalerite and the $\mathrm{CuInS}_{2}$ structure. Meanwhile, GaN has a structure similar to $\mathrm{ZnS}$ and is stable in the wurtzite phase. It was previously shown that the appearance of the sphalerite phase fragments within wurtzite GaN arose as a result of the introduction of a $\mathrm{Zn}$ impurity [16]. 
$\mathrm{ZnS}$ ores contain a large amount of various impurities. Namely, the traces of the following elements were found in samples of sphalerite: As, Sb, Bi, Sn, Ge, In, Mo, Pb, Cd, Fe, Mn, Cu, Hg, Ni, Co, Ag, Tl [17, 18]. Often, the mineral $\mathrm{ZnS}$ is accompanied by sulfides of iron, copper, lead and cadmium, which can both lower and increase the concentrations of individual impurity elements due to their redistribution [15, 17]. An impurity of $\mathrm{N}$ and $\mathrm{C}$ attributed to peptides was found in the biogenic samples of $\mathrm{ZnS}$ [19]. Many attempts have been undertaken to introduce a variety of dopants into synthetic ZnS: Mo [20], Ni [21], Sn [22], Cu [23, 24], Mn [23], N [25, 26], $\mathrm{C}[25]$, etc. The effect of these impurities on the formation of the $\mathrm{ZnS}$ crystal lattice remains often unknown and requires further research.

We have previously demonstrated that the $\mathrm{O}$ impurity replacing the $\mathrm{S}$ atoms has no significant effect on the mutual thermodynamic stability of the wurtzite and sphalerite phases, as well as of their mixed polytypes [27]. Here, the quantum chemistry method is employed to perform a comparative analysis of the chemical form, localization, and energy difference of the $\mathrm{N}$ impurity hosted at sphalerite and wurtzite lattices.

\section{Computational details}

The self-consistent-charge density-functional tight-binding method (DFTB) was used [28, 29]. This is an approximate method based on the density functional theory (DFT), enabling one to simulate the considerably large supercells. Hence, a low content of impurity within a periodic crystal can be studied, eliminating any spurious interaction between mirror images of the impurity atoms. A complete relaxation of the atomic positions and the optimization of lattice parameters were carried out, employing the periodic boundary conditions in $\Gamma$ point approximation as implemented in deMon program [30]. The 3OB-3-1 parametrization set of Slater-Koster parameters for description of all interatomic interactions was employed [31,32]. In addition, the main conclusions of our study were confirmed by calculations on the DFT-GGA level using the SIESTA software package [33].

Extended $3 a \times 3 a \times 3 a$ sphalerite and $3 a \times 3 \sqrt{v} a \times 3 c$ wurtzite cells consisting of 216 atoms were used as the basic models. The admixture of nitrogen was introduced into these cells after complete optimization of their geometry. Nitrogen was represented as one of three chemical species: an atom impurity N, molecular ammonia $\mathrm{NH}_{3}$ or ionic ammonium $\mathrm{NH}_{4}$. An impurity was hosted instead of the $\mathrm{Zn}$ or S single atoms as well as at the tetrahedral interstitial voids formed by four $\mathrm{Zn}$ or four $\mathrm{S}$ atoms. The octahedral voids in wurtzite were also considered. Different dispositions of the $\mathrm{H}$ atoms in the complex impurities $\left(\mathrm{NH}_{3}\right.$ and $\left.\mathrm{NH}_{4}\right)$ relative to a $\mathrm{ZnS}$ crystal lattice were also taken into account. In a number of models, the possibility of a $\mathrm{Zn}$ or S vacancy near an impurity was considered as well as the introduction of additional $\mathrm{H}$ atoms with the formation of $\mathrm{SH}$ groups near an impurity. Hereafter, the only relaxation of atomic positions in the designed models of defective crystals was carried out, while the values of translation vectors were maintained the same as for the pristine model of $\mathrm{ZnS}$. The thermodynamic stability of the models and the study of their electronic properties were performed after optimizing the model geometry.

Molecular dynamics (MD) simulations at the same DFTB level were used to confirm the kinetic stability of the most thermodynamically stable impurity defects. MD simulations have been performed using deMon code as for canonical ensembles (NVT). The annealing temperature was set to $100 \mathrm{~K}$ or $300 \mathrm{~K}$. In all simulations, the temperature was controlled by global Berendsen thermostat with the time constant $100 \mathrm{fs}$. The MD trajectories were collected during the time interval of $\sim 25 \mathrm{ps}$ with the time step $0.1 \mathrm{fs}$.

Rendering of atomic structures and MD trajectories was performed using VMD software [34].

\section{Results and discussion}

\subsection{Thermodynamic stability}

According to the results obtained by DFTB, sphalerite is slightly more stable than wurtzite by only 0.2 meV/atom. DFT calculations indicate a larger difference in energies (1.3 meV/atom), which is closer to the result $3.25 \mathrm{meV} /$ atom in [6]. In any case, such tiny differences may be attributed to a numerical error during computations.

An estimation of the thermodynamic stability of nitrogen impurity defects was performed according to the formation energies of nitrogen impurity defects in sphalerite or wurtzite $\left(E_{f}, \mathrm{eV}\right)$. The latter was calculated on the basis of DFTB simulations with respect to the pristine sphalerite crystal and the molecular species $\mathrm{S}_{8}, \mathrm{~N}_{2}, \mathrm{NH}_{3}$ and $\mathrm{H}_{2}$ according to the following formula:

$$
E_{f}=E_{\text {mod }} \cdot N-E_{Z n S} \cdot 2 N_{Z n}-E_{N H 3} \cdot 4 N_{N}-E_{S 8} \cdot\left(N_{S}-N_{Z n}\right)-E_{H 2} \cdot\left(N_{H}-3 N_{N}\right)
$$


where $E_{m o d}$ is the total energy of a model with a defect (eV/atom); $N$ is the total number of atoms in a model; $E_{Z n S}, E_{N H 3}, E_{S 8}, E_{H 2}$ are the total energies of sphalerite, $\mathrm{NH}_{3}, \mathrm{~S}_{8}$, and $\mathrm{H}_{2}$, respectively, given in the eV/atom; $N_{Z n}, N_{S}, N_{N}, N_{H}$ are the numbers of $\mathrm{Zn}, \mathrm{S}, \mathrm{N}$ and $\mathrm{H}$ atoms that make up the model $\left(N=N_{Z n}+N_{S}+N_{N}+N_{H}\right)$.

The four types of the most stable nitrogen impurity defects in $\mathrm{ZnS}$ are listed in Table 1 and their consequence turns out to be similar for both sphalerite and wurtzite. The fifth possible defect in the rank is different: in sphalerite it is the introduction of $\mathrm{NH}_{3}$ into the tetrahedral void formed by $\mathrm{Zn}$ atoms, while in wurtzite it is the introduction of $\mathrm{NH}_{3}$ into the octahedral void formed by $\mathrm{S}$ atoms. The most stable state of nitrogen in both $\mathrm{ZnS}$ polymorphs is the substitution of one $\mathrm{ZnS}$ unit by $\mathrm{NH}_{4} \mathrm{SH}$ unit. Its formation energy in sphalerite is found to be $0.54 \mathrm{eV}$ lower, than in wurtzite. All the following defects in Table 1 demonstrate the same pattern: the appearance of nitrogen impurity in sphalerite is energetically more beneficial than in wurtzite. However, the relative difference between the same types of defects in the $\mathrm{ZnS}$ polymorphs increases, approaching $1 \mathrm{eV}$.

TABLE 1. The rank of the most thermodynamically stable models of nitrogen impurity defects in $\mathrm{ZnS}$ crystals. DFTB calculations

\begin{tabular}{|c|c|c|c|}
\hline \multirow{2}{*}{ No. } & Defect description & \multicolumn{2}{c|}{ Defect formation energy, eV } \\
\cline { 3 - 4 } & & sphalerite & wurtzite \\
\hline I & replacement of a $\mathrm{ZnS}$ unit on $\mathrm{NH}_{4} \mathrm{HS}$ unit & -0.19 & +0.35 \\
\hline II & substitution of a $\mathrm{Zn}$ atom on $\mathrm{NH}_{4}$ & +0.72 & +1.75 \\
\hline III & replacement of a $\mathrm{ZnS}$ unit on single $\mathrm{NH}_{3} \mathrm{molecule}$ & +1.82 & +2.86 \\
\hline IV & substitution of a $\mathrm{Zn}$ atom on molecular $\mathrm{NH}_{3}$ & +1.82 & +2.88 \\
\hline
\end{tabular}

The difference between the formation energies of the same nitrogen impurity defects either in sphalerite or in wurtzite was confirmed by additional DFT calculations of the models I (Table 1) distinguished as the most stable defect type. The difference between the total energies of single defect in these models was estimated as $0.34 \mathrm{eV}$ according to the DFT results, which is well comparable to $0.54 \mathrm{eV}$ after DFTB calculation.

The simple substitution of an atom in a $\mathrm{ZnS}$ crystal for a single $\mathrm{N}$ atom impurity appears as the most unstable defect; e.g., the most stable model among these variants is the substituition of S atom by $\mathrm{N}$ with $E_{f} 6.33$ and 7.35 $\mathrm{eV}$ for sphalerite and wurtzite, respectively. The appearance of vacancies near an atomic $\mathrm{N}$ impurity leads only to further destabilization of the sphalerite and wurtzite lattices.

\subsection{Kinetic stability}

Molecular dynamics (MD) simulations were used to study the kinetic stability of three types of defects (I-III, Table 1) hosted in both sphalerite and wurtzite. The collection of the main results can be viewed at the links [35].

MD simulation of the model I from Table 1 was carried out at $300 \mathrm{~K}$ for sphalerite and at 100 and $300 \mathrm{~K}$ for wurtzite. This defect in the sphalerite crystal undergoes reversible changes: the $\mathrm{NH}_{4}$ group decomposes to $\mathrm{NH}_{3}$ molecule and $\mathrm{H}$ that forms the secondary $\mathrm{SH}$ group. The resulting $\mathrm{NH}_{3}$ molecule is coordinated on the nearest $\mathrm{Zn}$ atom. Afterwards, the ammonium recombines. The same defect in the wurtzite crystal undergoes similar processes at $300 \mathrm{~K}$, but ammonium recombination is not observed (Fig. 1). The defect is stable only at $100 \mathrm{~K}$, where the decay of $\mathrm{NH}_{4}$ is not observed.

Model II from Table 1 was investigated at 100 and $300 \mathrm{~K}$ for sphalerite and at $100 \mathrm{~K}$ for wurtzite. The defect in sphalerite is subject to reversible decomposition into $\mathrm{NH}_{3}$ and $\mathrm{SH}$ at $100 \mathrm{~K}$ and irreversible decomposition at $300 \mathrm{~K}$. Released $\mathrm{NH}_{3}$ molecule binds to one of the $\mathrm{Zn}$ atoms. Hence, the initial structure of this defect is kinetically unstable. This defect in wurtzite also disintegrates already at $100 \mathrm{~K}$.

MD simulations of model III from Table 1 at $T=300 \mathrm{~K}$ in both sphalerite and wurtzite demonstrate its kinetic stability. Molecular ammonia coordinates alternately at neighboring $\mathrm{Zn}$ atoms with dangling bonds.

Thus, single-type defects in wurtzite and sphalerite crystals exhibit similar kinetic stability. Replacement of a $\mathrm{ZnS}$ unit by a $\mathrm{NH}_{4} \mathrm{HS}$ unit or by a single $\mathrm{NH}_{3}$ molecule leads to the formation of a kinetically stable defect. The defect in which the $\mathrm{Zn}$ atom is replaced by single ammonium is kinetically unstable and prone to decomposition into $\mathrm{NH}_{3}$ molecule and $\mathrm{H}$ joining the $\mathrm{S}$ atom. 

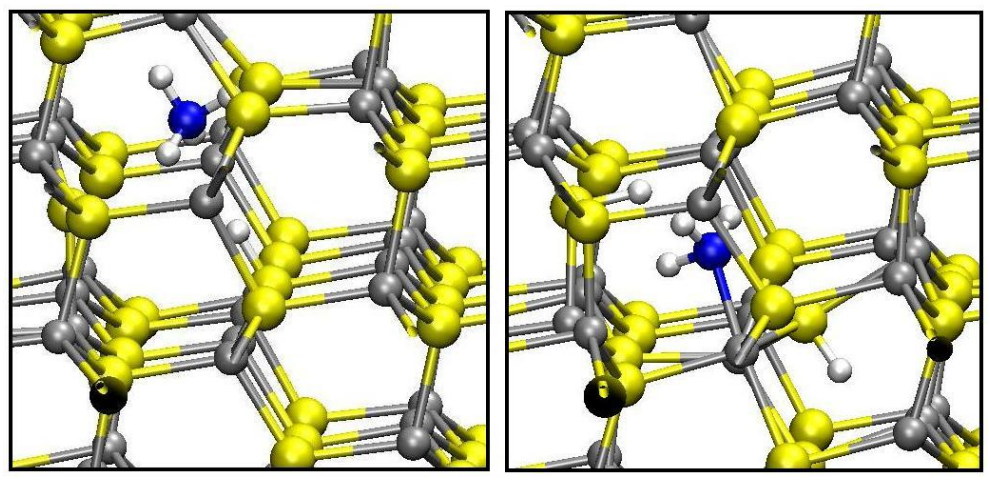

FIG. 1. Optimized model of the most thermodynamically stable nitrogen impurity defect (replacement of a $\mathrm{ZnS}$ unit on $\mathrm{NH}_{4} \mathrm{HS}$ unit) in wurtzite (on the left) and the results of its MD simulation at $T=300 \mathrm{~K}$ (on the right, see also the supplementary movies 1 and 2 [35]). Atom color coding: $\mathrm{Zn}$ - gray, $\mathrm{S}$ - yellow, $\mathrm{N}$ - blue, $\mathrm{H}$ - white

\subsection{Electronic properties}

The electronic properties of sphalerite and wurtzite $\mathrm{ZnS}$ pristine crystals, as well as crystals containing various nitrogen impurity defects, were investigated using the DFTB method. The plots of the electron densities of states (DOS) for wurtzite crystals are given in Fig. 2. DOSs of sphalerite crystal with similar types of defects have the same appearance.

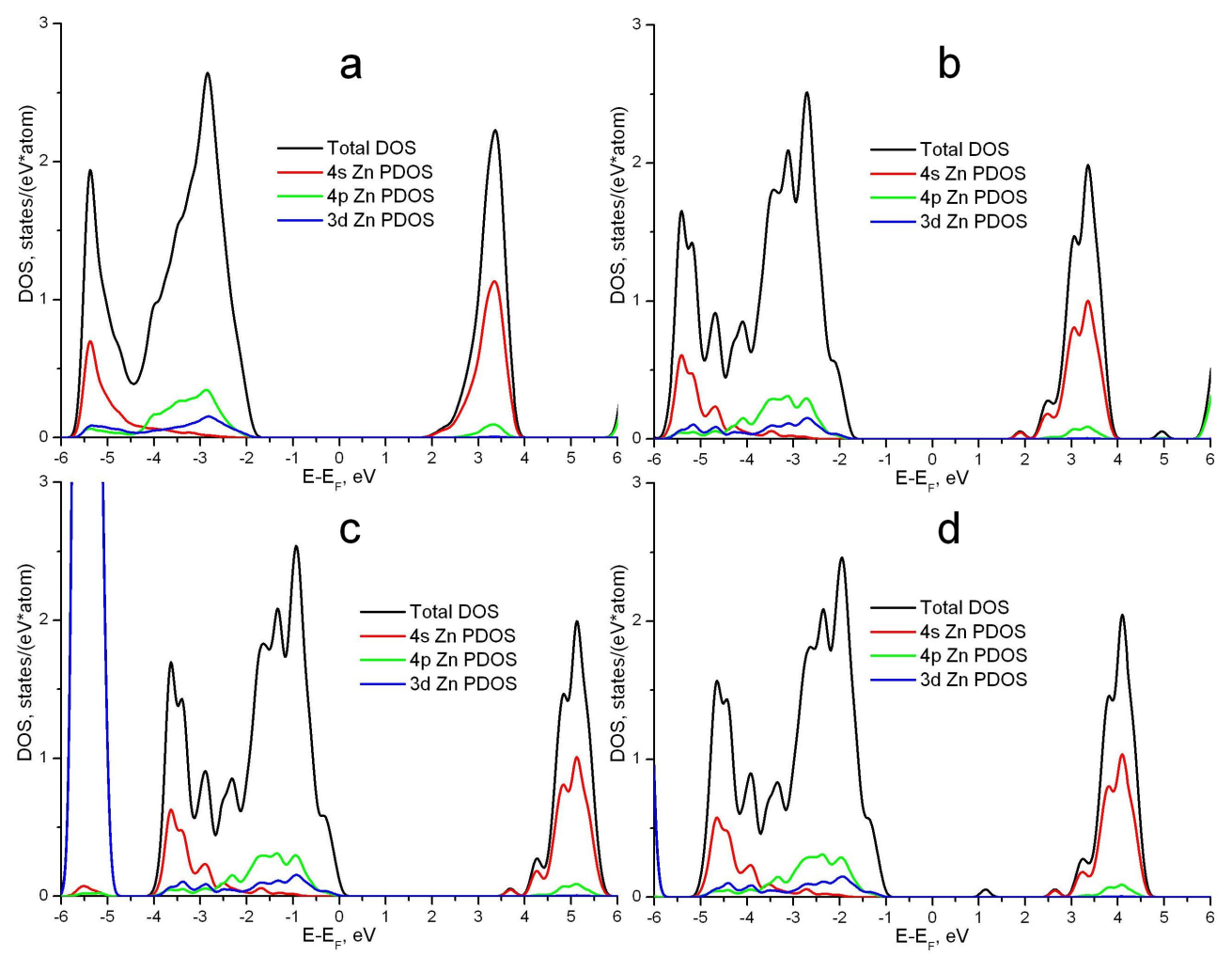

FIG. 2. Total and partial densities of states (DOS) for the wurtzite $\mathrm{ZnS}$ perfect crystal (a) and for the wurtzite $\mathrm{ZnS}$ crystal with different defects: replacement of a $\mathrm{ZnS}$ unit on $\mathrm{NH}_{4} \mathrm{HS}$ unit (b), substitution of a $\mathrm{Zn}$ atom on $\mathrm{NH}_{4}$ (c) substitution of a $\mathrm{Zn}$ atom on molecular $\mathrm{NH}_{3}$ (d) (models I, II and IV in Table 1, respectively)

The sphalerite and wurtzite are semiconductors with the fundamental band gaps $\left(E_{g}\right)$ equal to 3.73 and $3.80 \mathrm{eV}$, respectively (Fig. 2(a)). These values are in good agreement with the experimental data of 3.68 and $3.91 \mathrm{eV}$ [36]. 
The valence band of both crystals is formed predominantly by $\mathrm{S} 3 p$ states, and the bottom of conduction band by $\mathrm{Zn} 4 s$ states.

Replacement of single $\mathrm{ZnS}$ unit by $\mathrm{NH}_{4} \mathrm{HS}$ (model I, Table 1) leads to the splitting of a part of the localized $\mathrm{Zn} 4 s$ states from the bottom of conduction band (Fig. 2(b)). Similar changes in DOS occur for the introduction of the $\mathrm{NH}_{3}$ molecule at the tetrahedral void formed by four $\mathrm{Zn}$ and for the substitution of a $\mathrm{ZnS}$ unit on single $\mathrm{NH}_{3}$ molecule (model III, Table 1).

Substitution of a $\mathrm{Zn}$ atom on $\mathrm{NH}_{4}$ (model II from Table 1) leads to a shift of the Fermi level $\left(E_{F}\right)$ to the edge of the valence band (Fig. 2(c)), which is characteristic for a $p$-type semiconductor. DOS near $E_{F}$ are formed predominantly by $\mathrm{S} 3 p$ states. Similar changes in DOS can be observed for other unstable defect - the replacement of one atom $\mathrm{S}$ by atom $\mathrm{N}$ which agrees with the earlier DFT study [37].

Substitution of a $\mathrm{Zn}$ atom on $\mathrm{NH}_{3}$ molecule leads to a new state located within the fundamental band gap (Fig. 2(d)). This state is formed mainly by S3p and N2p orbitals. Orbitals' visualization of the electron wave function isosurfaces reveals that this state is strongly localized (Fig. 3), hence, it cannot lead to a decrease of the value $E_{g}$.

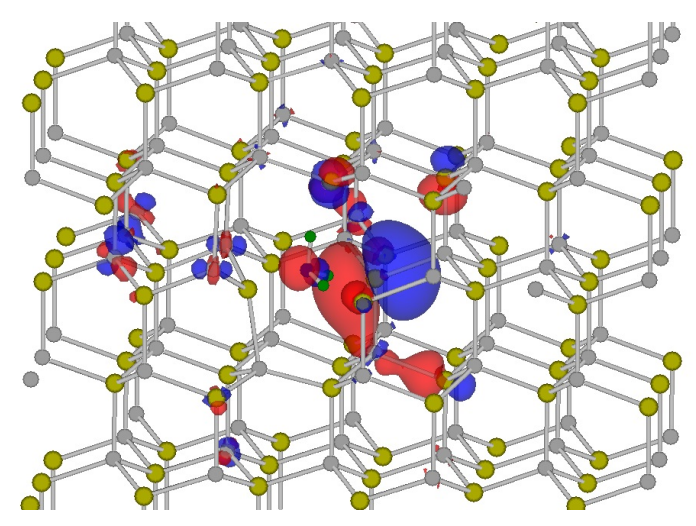

FIG. 3. Orbitals visualization of the electron wave function isosurface for the states located within the fundamental band gap of the model IV (Table 1). Color coding: $\mathrm{Zn}$ - gray, $\mathrm{S}$ - yellow, $\mathrm{N}$ - blue, $\mathrm{H}$ - green

\section{Summary}

A comparative analysis of the most stable form and the localization of nitrogen impurities in sphalerite and wurtzite $\mathrm{ZnS}$ crystals has been carried out using DFTB method. The effect of impurities on polymorphic equilibrium in $\mathrm{ZnS}$ is considered. It was found that the most stable type of $\mathrm{N}$ impurity, irrespective of the $\mathrm{ZnS}$ polymorph, is the substitution of one $\mathrm{ZnS}$ unit on $\mathrm{NH}_{4} \mathrm{SH}$. However, the thermodynamic stability of this defect depends on the $\mathrm{ZnS}$ polymorphism. The rise of this defect in the $\mathrm{ZnS}$ lattice leads to the removal of enthalpy degeneracy of wurtzite and sphalerite in favor of the latter. Perhaps, this effect may also be caused by other impurities, which would explain the wider distribution of sphalerite in nature.

Various types of $\mathrm{N}$ impurities modify differently the band gap of $\mathrm{ZnS}$ polymorphs. The most stable substitutional defect - the $\mathrm{NH}_{4} \mathrm{SH}$ unit - may lead to a slight narrowing of the band gap.

\section{Acknowledgements}

The work was financially supported by the Russian Science Foundation (Grant No. 17-79-20165).

\section{References}

[1] Posfai M., Dunin-Borkowski R.E. Sulfides in Biosystems. Rev. Mineral. Geochem., 2006, 61, P. 679-714.

[2] Xu J., Murayama M., et al. Highly-defective nanocrystals of ZnS formed via dissimilatory bacterial sulfate reduction: A comparative study with their abiogenic analogues. Geochim. Cosmochim. Acta, 2016, 180, P. 1-14.

[3] Zhang H., Huang F., Gilbert B., Banfield J.F. Molecular Dynamics Simulations, Thermodynamic Analysis, and Experimental Study of Phase Stability of Zinc Sulfide Nanoparticles. J. Phys. Chem. B, 2003, 107, P. 13051-13060.

[4] Qadri S.B., Skelton E.F., et al. Size-induced transition-temperature reduction in nanoparticles of ZnS. Phys. Rev. B, 1999, 60 (13), P. 9191-9193.

[5] Lin P.-C., Hua C.C., Lee T.-C. Low-temperature phase transition of ZnS: The critical role of ZnO. J. Solid Chem., 2012, 194, P. $282-285$.

[6] Boutaiba F., Belabbes A., Ferhat M., Bechstedt F. Polytypism in ZnS, ZnSe, and ZnTe: First-principles study. Phys. Rev. B, 2014, 89, 245308. 
[7] Zhao Y., Zhang Y., et al. Low-Temperature Synthesis of Hexagonal (Wurtzite) ZnS Nanocrystals. J. Am. Chem. Soc., 2004, 126, P. 68746875.

[8] Yin L.W., Bando Y., et al. Self-assembled highly faceted wurtzitetype ZnS single-crystalline nanotubes with hexagonal cross-sections. Adv. Mater., 2005, 17, P. 1972-1977.

[9] La Porta F.A., Andres J., et al. Sphalerite versus wurtzite ZnS nanoparticles: control of the phase and optical properties by tetrabutylammonium hydroxide. Phys. Chem. Chem. Phys., 2014, 16, P. 20127-20137.

[10] Huang F., Zhang H., Banfield J.F. The Role of Oriented Attachment CityplaceCrystal Growth in Hydrothermal Coarsening of Nanocrystalline ZnS. J. Phys. Chem. B, 2003, 107 (38), P. 10470-10475.

[11] Luther G.W., Theberge S.M., Rickard D.T. Evidence for aqueous clusters as intermediates during zinc sulfide formation. Geochim. Cosmochim. Acta, 1999, 63, P. 3159-3169.

[12] Zhang H., Huang F., Gilbert B., Banfield J.F. Molecular Dynamics Simulations, Thermodynamic Analysis, and Experimental Study of Phase Stability of Zinc Sulfide Nanoparticles. J. Phys. Chem. B, 2003, 107, P. 13051-13060.

[13] Vasil'ev V.I. New data on the composition of metacinnabar and Hg-sphalerite with an isomorphous Cd admixture. Russian Geology and Geophysics, 2011, 52, P. 701-708.

[14] Nitta E., Kimata M., et al. Crystal chemistry of $\mathrm{ZnS}$ minerals formed as high-temperature volcanic sublimates: matraite identical with sphalerite. J. Mineral. Petrol. Sci., 2008, 103, P. 145-151.

[15] Chaplygin I.V., Mozgova N.N., et al. Minerals of the system ZnS-CdS from fumaroles of the Kudriavy volcano, Iturup Island, Kuriles, Russia. Can. Mineral., 2007, 45, P. 709-722.

[16] Yang B., Liu B., et al. Zn-dopant dependent defect evolution in GaN nanowires. Nanoscale, 2015, 7, P. 16237-16245.

[17] George L.L., Cook N.J., Ciobanu C.L. Partitioning of trace elements in co-crystallized sphalerite-galena-chalcopyrite hydrothermal ores. Ore Geol. Rev., 2016, 77, P. 97-116.

[18] Pfaff K., Koenig A., et al. Trace and minor element variations and sulfur isotopes in crystalline and colloform ZnS: Incorporation mechanisms and implications for their genesis. Chem. Geol., 2011, 286, P. 118-134.

[19] Moreau J.W., Weber P.K., et al. Extracellular Proteins Limit the Dispersal of Biogenic Nanoparticles. Science, 2007,316, P. 1600-1603.

[20] Zhang R., Du B., et al. Molybdenum-doped ZnS sheets with dominant $\{111\}$ facets for enhanced visible light photocatalytic activities. $J$. Colloid Interface Sci., 2017, 507, P. 200-208.

[21] Kudo A., Sekizawa M. Photocatalytic $\mathrm{H}_{2}$ evolution under visible light irradiation on Ni-doped ZnS photocatalyst, Chem. Commun., 2000, 15, P. 1371-1372.

[22] Kumar K.C., Rao N.M., Kaleemulla S., Rao G.V. Structural, optical and magnetic properties of Sn doped ZnS nano powders prepared by solid state reaction. Physica B: Condens. Matter, 2017, 522, P. 75-80.

[23] Patel P.C., Ghosh S., Srivastava P.C. Effect of impurity concentration on optical and magnetic properties in ZnS: Cu nanoparticles. Physica E: Low-dimens. Syst. Nanostruct., 2017, 93, P. 148-152.

[24] Al-Jawad S.M.H., Ismail M.M. Characterization of $\mathrm{Mn}, \mathrm{Cu}$, and (Mn, Cu) co-doped ZnS nanoparticles. J. Opt. Techn., 2017, 84, P. 495-499.

[25] Muruganandham M., Kusumoto Y. Synthesis of N, C codoped hierarchical porous microsphere ZnS as a visible light-responsive photocatalyst. J. Phys. Chem. C, 2009, 113, P. 16144-16150.

[26] Popov I.S., Kozhevnikova N.S., et al. Nitrogen-doped ZnS nanoparticles: Soft-chemical synthesis, EPR statement and quantum-chemical characterization. Mater. Chem. Phys., 2018, 215, P. 176-182.

[27] Popov I.S., Vorokh A.S., Enyashin A.N. Stability and electronic properties of oxygen-doped ZnS polytypes: DFTB study. Chem. Phys., 2018, 510, P. 70-76.

[28] Seifert G. Tight-binding density functional theory: an approximate Kohn-Sham DFT scheme. J. Phys. Chem. A, 2007, 111, P. 5609-5613.

[29] Frauenheim T., Seifert G., et al. A Self-consistent charge density-functional based tight-binding method for predictive materials simulations in physics, chemistry and biology. Phys. Status Solidi B, 2000, 217, P. 41-62.

[30] Koster A.M., Flores R., et al. Program deMon (version 1.1.0). National Research Council, Ottawa, Canada, 2003.

[31] Gaus M., Lu X., Elstner M., Cui Q. Parameterization of DFTB3/3OB for sulfur and phosphorus for chemical and biological applications. J. Chem. Theory Comput., 2014, 10, P. 1518-1537.

[32] Lu X., Gaus M., Elstner M., Cui Q. Parametrization of DFTB3/3OB for magnesium and zinc for chemical and biological applications. J. Phys. Chem. B, 2015, 119, P. 1062-1082.

[33] Soler J.M., Artacho E., et al. The SIESTA method for ab initio order-N materials simulation. J. Phys. Condens. Matter, 2002, 14 (11), P. $2745-2779$.

[34] Humphrey W., Dalke A., Schulten K. VMD: Visual molecular dynamics. J. Mol. Graphics, 1996, 14, P. 33-38.

[35] Videolinks: (Movie 1) Defect model I in wurtzite. URL: https://youtu.be/mr9ZP15Pj8Y. (Movie 2) Defect model I in sphalerite. URL: https://youtu.be/dEy_K7hbN3c. (Movie 3) Defect model II in wurtzite. URL: https://youtu.be/7yHPhP6EEhA. (Movie 4) Defect model II in sphalerite. URL: https://youtu.be/q_HVyrS8QeQ. (Movie 5) Defect model III in wurtzite. URL: https://youtu.be/04-pDu1IruE. (Movie 6) Defect model III in sphalerite. URL: https://youtu.be/_OHRE-Pfh3g.

[36] Derek W.P. The semiconductors-information web-site. URL: http://www.semiconductors.co.uk/propiivi5410.htm (on the date $05.12 .2018)$.

[37] Long R., English N.J. Magnetic properties of first-row element-doped ZnS semiconductors: A density functional theory investigation. Phys. Rev. B, 2009, 80, 115212 . 